\title{
Do Biologic-treated Psoriatic Arthritis Patients with Spondylitis Respond Differently with or without Concomitant Methotrexate from Patients without Spondylitis?
}

How effective is methotrexate (MTX) in psoriatic arthritis (PsA)? Should we use MTX in combination with biologic therapy in PsA? Does MTX increase therapeutic benefit when used in combination with biologics, either because of its own immunomodulatory effect or its ability to decrease immunogenicity to biologics? Or does MTX not provide additional benefit over and above the biologic agent? Instead, does it contribute only problems from a tolerability and safety perspective?

Despite the fact that MTX is the most commonly used immunomodulatory drug in PsA, these questions still have not been satisfactorily answered. A variety of studies sheds light on these questions, but uncertainty remains.

In this issue of The Journal, Behrens, et al study a large registry cohort in Germany to attempt to address a corollary question ${ }^{1}$. Knowing that MTX is not effective in treating the spinal symptoms of ankylosing spondylitis ${ }^{2}$, they ask the following question: In a cohort of patients with PsA, about half treated with adalimumab (ADA) monotherapy and half with concomitant MTX, if PsA subjects with spondylitis symptoms are analyzed separately from those with peripheral inflammatory musculoskeletal symptoms only, is there any difference in response to 2 years of treatment based on MTX background?

There have been few placebo-controlled trials to establish the efficacy of MTX in PsA using the low-dose MTX regimen used for the treatment of rheumatoid arthritis (RA) and psoriasis. Neither Willkens, et al nor Kingsley, et al were able to demonstrate benefit of MTX over placebo as assessed by arthritis measures used at the times of those trials, although patient global assessment and some skin measures showed modest improvement ${ }^{3,4,5}$. Arguably, these were not fair trials in that the first trial studied few patients and included a low-dose arm (7.5 mg as well as $15 \mathrm{mg}$ ) whereas the more recent trial had significant patient dropout and included many patients with relatively low disease severity ${ }^{5}$. Neither trial assessed radiographic outcomes, so ability to have an effect on structural damage was not assessed. On the other hand, an open-label study comparing MTX with MTX plus infliximab (IFX) in a relatively early PsA cohort demonstrated good American College of Rheumatology (ACR) response in the MTX monotherapy arm: ACR 20/50/70 responses of $67 / 40 / 19 \%$, respectively ${ }^{6}$. Although these results need to be taken with a grain of salt since this was an open-label study, nonetheless they give some support to the clinically observed positive responses of peripheral arthritis seen in some patients with PsA in routine clinical practice. In any case, firm evidence is lacking from a placebo-controlled trial, adequately powered and dosed in an appropriate PsA cohort, leaving us in a state of uncertainty about the effect of MTX monotherapy in PsA.

Can MTX, when used in combination with a biologic agent, provide additional benefit beyond the benefit achieved from the biologic alone? This question has been addressed in depth in the context of RA. Numerous studies have suggested that addition of MTX to biologic therapy will yield superior clinical and radiographic outcomes compared to biologic or MTX monotherapy ${ }^{7,8}$. Thus, combination biologic plus MTX therapy has become standard practice in RA. Evidence to answer this question in PsA is lacking. In the phase III trials of various biologics, including anti-tumor necrosis factor, anti-interleukin 12 (IL-12)/IL-23, and anti-IL-17, which included biologic monotherapy and biologic combined with MTX arms, the combination of biologic with MTX did not yield greater benefit than biologic monotherapy in arthritis and skin responses $9,10,11,12,13$. Because the patients in these trials were considered to be MTX inadequate responders, it is not appropriate to judge the potential value of MTX combination from these studies.

An indirect way to assess such value is to look at survival

See Does MTX with ADA influence treatment outcomes in PsA? page 632

Personal non-commercial use only. The Journal of Rheumatology Copyright @ $\odot 2016$. All rights reserved. 
curves of clinical registry subjects using biologic monotherapy versus combination with MTX. This has been studied in the Norwegian, Swedish, and CORRONA PsA registries in relation to etanercept (ETN), ADA, and IFX ${ }^{14,15,16}$. IFX survival is more prolonged when MTX is combined compared to IFX monotherapy. This does not appear to be the case with ETN and is possibly not the case with ADA. One explanation for these observations, at least partially, is that more prolonged survival taking IFX plus MTX denotes more sustained effectiveness, whereas ETN and possibly ADA can have sustained effectiveness in monotherapy form. This could be the result of a direct added benefit of MTX in those patients, of reduced immunogenicity against IFX with the use of MTX, or both. One expects a certain amount of immunogenicity against IFX given its chimeric antibody property. However, it is important to keep in mind that this is clinical registry data, so firm conclusions cannot be drawn. Vogelzang, et al have studied the effect of anti-drug antibodies against ADA in PsA and demonstrated that the presence of such antibodies was associated with lower serum levels of ADA and with lower treatment benefit; moreover, concomitant use of MTX could reduce anti-drug antibody formation ${ }^{17,18}$. These findings suggest that ADA effectiveness in some patients can be increased through use of MTX to inhibit anti-drug antibody formation. These findings have not yet translated into being readily useful in clinical practice, i.e., in terms of being able to easily and reliably measure drug antibody levels.

In this issue of The Journal, Behrens, et al have attempted to determine whether PsA patients with inflammatory spine disease due to PsA have a different response when ADA is combined with MTX versus patients with purely peripheral arthritis ${ }^{1}$. In large cohort studies, inflammatory spondylitis involving the spine and/or sacroiliac joints is suggested to occur in about $40 \%$ of patients with $\mathrm{PsA}^{19}$. In PsA clinical trials, spine involvement and spine response to treatment is not usually assessed because patients with spondylitis are in the minority, its presentation can be quite variable, and accurate spondylitis diagnosis and assessment of response would require extensive and expensive magnetic resonance imaging (MRI), as well as clinical assessment. Thus, to establish treatment recommendations for PsA spondylitis, international groups such as the Group for Research and Assessment of Psoriasis and Psoriatic Arthritis (GRAPPA) use data from ankylosing spondylitis (AS) trials as a surrogate for spondylitis response in $\mathrm{Ps}^{20}$. In AS studies, none of the traditional oral disease-modifying antirheumatic drugs (DMARD), including MTX, have demonstrated efficacy in the spinal manifestations of the disease. Thus, we generally assume, although it is not proven, that similar findings would be found in PsA spondylitis. If this is the case, and a substantial percentage of PsA patients in a cohort have spondylitis, then when studying whether combination MTX and biologic is more effective than biologic alone, it is possible that nonresponse in the spine could diminish the ability to discriminate between patients using MTX and those who are not.

The Behrens study was a non-interventional study involving 355 German centers from whom 1455 patients with PsA being treated with ADA were analyzed. Of these patients, $20 \%$ were considered to have axial involvement, i.e., PsA spondylitis, and the remaining $80 \%$ constituted the "peripheral" group. In both groups of patients, about 55\% were treated with ADA monotherapy and the remainder with concomitant MTX.

An important limitation of the study, acknowledged by the authors, was that determination of axial involvement was based on the investigator's clinical judgment - not on objective markers such as radiography or MRI. Presumably, the investigator determined that the patient had inflammatory back pain - even this was not specified - or had some other feature that allowed discrimination between inflammatory spondylitis and degenerative or mechanical back pain (a difficult distinction to make in the absence of objective markers). However, it is somewhat reassuring that the prevalence of spondylitis was just $20 \%$ in this study, i.e., not higher than the expected prevalence.

The key message from their study was that ADA treatment led to significant improvement in measures of PsA other than spondylitis, in both the spondylitis-present and spondylitis-absent group, and there was no difference in the degree of response in the MTX-present as compared to the MTX-absent groups when multiple regression analyses were applied to account for baseline differences between groups. Safety and tolerability was also similar between MTX-present and MTX-absent groups. A further analysis of patients who changed therapy (adding or dropping MTX) during the observation period did not show an effect of this change on treatment response, suggesting that the lack of difference in response was due to differential dropout. Total withdrawals occurred in $32 \%$ of spondylitis-present and $24 \%$ of spondylitis-absent patients and were the same regardless of concomitant MTX therapy, as was the Kaplan-Meier survival curve for ADA persistence.

Unlike the paradigm of treatment in RA, wherein the combination of biologic agent plus MTX is considered the optimal approach to therapy to provide further immunomodulation and prevention of immunogenicity, in PsA the picture is different. Controlled trials, albeit of insufficient strength and quality, have failed to show clear benefit of MTX compared to placebo. Concomitant MTX does not clearly affect ETN or ADA persistence, but does affect IFX persistence in PsA clinical registries. Now the Behrens study provides analysis of the subsets of PsA patients with and without spondylitis treated with $\mathrm{ADA}$, and here, too, concomitant MTX does not appear to affect treatment response, adverse effects, or persistence. On the other hand, data from Vogelzang demonstrate that MTX can reduce ADA

Personal non-commercial use only. The Journal of Rheumatology Copyright @ 2016 . All rights reserved. 
anti-drug antibodies, and thus could be helpful in the subgroup of patients in whom such antibodies will affect therapeutic response - if we can figure out who that will be. So there is no clear-cut guidance; and not, as in RA, a clearly positive role for MTX in most cases.

The reader will appreciate that we may get some guidance from a study now under way. Eight hundred and forty patients with PsA who are naive to MTX are being randomized to MTX alone, ETN alone, and the combination of these 2 agents. Endpoints will include measures such as ACR response, achievement of minimal disease activity, enthesitis, dactylitis, skin and nail response, and radiographic outcomes. Such a substantial prospective trial, as well as ongoing mining of large clinical cohorts and registries, as reflected in the Behrens paper, may provide more sound guidance regarding biologic monotherapy versus combination for optimal clinical management of patients with PsA.

\section{PHILIP J. MEASE, MD,}

Swedish Medical Center,

Division of Rheumatology Research;

University of Washington School of Medicine, Seattle, Washington, USA.

Address correspondence to Dr. P.J. Mease, Seattle Rheumatology Associates, 601 Broadway, Suite 600, Seattle, Washington 98122, USA.

E-mail: pmease@philipmease.com

\section{REFERENCES}

1. Behrens F, Koehm M, Arndt U, Wittig BM, Greger G, Thaci D, et al. Does concomitant methotrexate with adalimumab influence treatment outcomes in patients with psoriatic arthritis? Data from a large observational study. J Rheumatol 2016;43:632-9.

2. Haibel H, Sieper J. Use of methotrexate in patients with ankylosing spondylitis. Clin Exp Rheumatol 2010;28:S128-31.

3. Willkens RF, Williams HJ, Ward JR, Egger MJ, Reading JC, Clements PJ, et al. Randomized, double-blind, placebo controlled trial of low-dose pulse methotrexate in psoriatic arthritis. Arthritis Rheum 1984;27:376-81.

4. Kingsley GH, Kowalczyk A, Taylor H, Ibrahim F, Packham JC, McHugh NJ, et al. A randomized placebo-controlled trial of methotrexate in psoriatic arthritis. Rheumatology 2012;51:1368-77.

5. Mease PJ. Spondyloarthritis: Is methotrexate effective in psoriatic arthritis? Nat Rev Rheumatol 2012;8:251-2.

6. Baranauskaite A, Raffayova H, Kungurov NV, Kubanova A, Venalis A, Helmle L, et al. Infliximab plus methotrexate is superior to methotrexate alone in the treatment of psoriatic arthritis in methotrexate-naive patients: the RESPOND study. Ann Rheum Dis 2012;71:541-8.

7. Breedveld FC, Weisman MH, Kavanaugh AF, Cohen SB, Pavelka $\mathrm{K}$, van Vollenhoven $\mathrm{R}$, et al. The PREMIER study: A multicenter, randomized, double-blind clinical trial of combination therapy with adalimumab plus methotrexate versus methotrexate alone or adalimumab alone in patients with early, aggressive rheumatoid arthritis who had not had previous methotrexate treatment. Arthritis Rheum 2006;54:26-37.
8. van der Heijde D, Klareskog L, Rodriguez-Valverde V, Codreanu C, Bolosiu H, Melo-Gomes J, et al. Comparison of etanercept and methotrexate, alone and combined, in the treatment of rheumatoid arthritis: two-year clinical and radiographic results from the TEMPO study, a double-blind, randomized trial. Arthritis Rheum 2006;54:1063-74

9. Mease P, Kivitz A, Burch F, Siegel E, Cohen S, Ory P, et al. Etanercept treatment of psoriatic arthritis: safety, efficacy, and effect on disease progression. Arthritis Rheum 2004;50:2264-72.

10. Mease PJ, Gladman DD, Ritchlin CT, Ruderman EM, Steinfeld SD, Choy EH, et al. Adalimumab for the treatment of patients with moderately to severely active psoriatic arthritis: results of a double-blind, randomized, placebo-controlled trial. Arthritis Rheum 2005;52:3279-89.

11. Antoni C, Krueger GG, de Vlam K, Birbara C, Beutler A, Guzzo C, et al. Infliximab improves signs and symptoms of psoriatic arthritis: results of the IMPACT 2 trial. Ann Rheum Dis 2005;64:1150-7.

12. Mease PJ, Fleischmann R, Deodhar AA, Wollenhaupt J, Khraishi M, Kielar D, et al. Effect of certolizumab pegol on signs and symptoms in patients with psoriatic arthritis: 24-week results of a Phase 3 double-blind randomised placebo-controlled study (RAPID-PsA). Ann Rheum Dis 2014;73:48-55.

13. Kavanaugh A, McInnes I, Mease P, Krueger GG, Gladman D, Gomez-Reino J, et al. Golimumab, a new human tumor necrosis factor alpha antibody, administered every four weeks as a subcutaneous injection in psoriatic arthritis: Twenty-four-week efficacy and safety results of a randomized, placebo-controlled study. Arthritis Rheum 2009;60:976-86.

14. Fagerli KM, Lie E, van der Heijde D, Heiberg MS, Lexberg AS, Rodevand E, et al. The role of methotrexate co-medication in TNF-inhibitor treatment in patients with psoriatic arthritis: results from 440 patients included in the NOR-DMARD study. Ann Rheum Dis 2014;73:132-7.

15. Kristensen LE, Gulfe A, Saxne T, Geborek P. Efficacy and tolerability of anti-tumour necrosis factor therapy in psoriatic arthritis patients: results from the South Swedish Arthritis Treatment Group register. Ann Rheum Dis 2008;67:364-9.

16. Mease PJ, Collier DH, Saunders KC, Li G, Kremer JM, Greenberg JD. Comparative effectiveness of biologic monotherapy versus combination therapy for patients with psoriatic arthritis: results from the Corrona registry. RMD Open 2015;1:e00181.

17. Vogelzang EH, Kneepkens EL, Nurmohamed MT, van Kuijk AW, Rispens T, Wolbink G, et al. Anti-adalimumab antibodies and adalimumab concentrations in psoriatic arthritis; an association with disease activity at 28 and 52 weeks of follow-up. Ann Rheum Dis 2014; 73:2178-82.

18. Vogelzang EH, Pouw MF, Nurmohamed M, Kneepkens EL, Rispens $\mathrm{T}$, Wolbink GJ, et al. Adalimumab trough concentrations in patients with rheumatoid arthritis and psoriatic arthritis treated with concomitant disease-modifying antirheumatic drugs. Ann Rheum Dis 2015;74:474-5.

19. Gladman DD. Clinical features and diagnostic considerations in psoriatic arthritis. Rheum Dis Clin North Am 2015;41:569-79.

20. Coates LC, Kavanaugh A, Mease PJ, Soriano ER, Acosta Felquer ML, Armstrong AW, et al. Group for research and assessment of psoriasis and psoriatic arthritis: treatment recommendations for psoriatic arthritis 2015. Arthritis Rheumatol 2016 Jan 8 (E-pub ahead of print).

J Rheumatol 2016;43:471-3; doi:10.3899/jrheum.160046 